\title{
Ocorrência de depressão relacionada ao trabalho no interior de Minas Gerais
}

\author{
Occurrence of work-related depression in the interior of Minas Gerais \\ Ocurrencia de depresión relacionada con el trabajo en el interior de Minas Gerais
}

Recebido: 30/06/2021 | Revisado: 10/07/2021 | Aceito: 13/07/2021 | Publicado: 24/07/2021

Aldo Matos
ORCID: https://orcid.org/0000-0001-8924-5116
Universidade de Uberaba, Brasil
E-mail: aldo.matos@ uniube.br
Marciana Fernandes Moll
Universidade de Uberaba, Brasil
ORCID: https:/orcid.org/0000-003-4794-4255
E-mail: marciana.fernandes@ uniube.br
Paulo Sérgio Mendes Oliveira
ORCID: https://orcid.org/ 0000-0002-6724-6385
Universidade de Uberaba, Brasil
E-mail paulllosergyo@gmail.com
Raíssa Beatriz Araújo Prata Galdino
ORCID: https://orcid.org/0000-0002-7679-1166
Universidade de Uberaba, Brasil
E-mail: raissaaraujo886@ @mail.com
Wilson Carlos Barbosa de Almeida
ORCID: https://orcid.org/0000-0001-5159-1822
Universidade de Uberaba, Brasil
E-mail: wilson.almeida@ @otmail.com
Josiana Dias Silva Trajano
Universidade de Uberaba, Brasil
E-mail: josiana_dias@yahoo.com.br
Anna Neri Batista da Silva
ORCID: https://orcid.org/0000-0003-4570-4362
ORCID: https://orcid.org/0000-0001-7819-6021
E-mail: nericabs@ @hotmail.com

\section{Resumo}

Descrever a ocorrência da depressão relacionada ao trabalho no interior de Minas Gerais e investigar a associação entre depressão relacionada ao trabalho e sexo, idade, situação no mercado de trabalho, tempo de trabalho na ocupação e a categoria da ocupação. Estudo retrospectivo, descritivo e quantitativo, com análise de todas as notificações de depressão relacionadas ao trabalho acompanhadas em um serviço público de saúde do trabalhador, no período de janeiro de 2013 a dezembro de 2019. Os dados coletados foram armazenados em planilhas do Excel, em dupla entrada com checagem. Para a análise dos dados, utilizou-se o programa Bioestat 5.0 e para as variáveis categóricas utilizou-se o teste Qui-quadrado $(\alpha=0,05)$. Houve um predomínio do sexo feminino. Encontrou-se associação da depressão grave com o sexo feminino, com a situação no mercado de trabalho e com a faixa etária. Não houve associação da doença à categoria profissional. Faz-se necessário investir em estratégias de promoção e proteção da saúde mental no ambiente de trabalho, sobretudo entre as mulheres que tendem a ter jornadas de trabalho no âmbito profissional e familiar.

Palavras-chave: Transtorno depressivo; Saúde do trabalhador; Saúde mental.

\begin{abstract}
To describe the occurrence of work-related depression notified in the interior of Minas Gerais and investigate the association between work-related depression and gender, age, situation in the labor market, working time in occupation and occupation category. A retrospective, descriptive and quantitative study, with analysis of all notifications of work-related depression accompanied in a public service specialized in worker health, from January 2013 to December 2019. The collected data was stored in Excel spreadsheets, in double entry with checking. For the data analysis, the Bioestat 5.0 program was used and for the categorical variables, the Chi-square test was used ( $\alpha=$ 0.05). There was a predominance of female notifications. There was an association of depression with the female gender, specifically severe depression, with the situation in the work market and age group. There was no association of the disease to the occupational category. It is necessary to invest in strategies to promote and protect mental health in the work environment, especially among women who tend to have professional and family work days.
\end{abstract}

Keywords: Depressive disorder; Occupational health; Mental health. 


\begin{abstract}
Resumen
Describa la ocurrencia de depresión relacionada con el trabajo en el interior de Minas Gerais e investigar la asociación entre la depresión relacionada con el trabajo y el género, la edad, la situación en el mercado laboral, tiempo de trabajo en la ocupación y la categoría de ocupación. Estudio retrospectivo, descriptivo y cuantitativo, con análisis de todos los informes de depresión relacionada con el trabajo en un servicio público de salud de los trabajadores desde enero de 2013 hasta diciembre de 2019. Los datos recopilados se almacenaron en hojas de cálculo de Excel, doble entrada con cheque. El programa Bioestat 5.0 se utilizó para el análisis de datos y para las variables categóricas, se utilizó la prueba chi-cuadrada $(\alpha$ a 0,05$)$.Había un predominio de las hembras. Se encontró una asociación de depresión severa con las mujeres, la situación en el mercado laboral y el grupo de edade. No hubo asociación de la enfermedad con la categoría profesional. Es necesario invertir en estrategias para la promoción y protección de la salud mental en el lugar de trabajo, especialmente entre las mujeres que tienden a tener horas de trabajo en el trabajo y la familia.
\end{abstract}

Palabras clave: Trastorno depresivo; Salud laboral; Salud mental.

\title{
1. Introdução
}

O trabalho faz parte do cotidiano dos seres humanos e quando administrado satisfatoriamente é terapêutico. Contudo, o capitalismo vem transformando o trabalho em um dispositivo de acúmulo de bens materiais e isso tende a gerar agravos na saúde do trabalhador (Teixeira \& Vieira, 2018). Na atualidade, o desenvolvimento tecnológico e financeiro gera cobranças e competitividade acarretando aumento de transtornos mentais relacionadas ao trabalho (Vitorino et al., 2018). Acrescenta-se que outros fatores também predispõem aos referidos transtornos, são eles: sobrecarga, jornadas excessivas, baixa remuneração, múltiplos vínculos empregatícios, entre outros (Fernandes et al., 2018).

Diante dessa realidade, a saúde do trabalhador vem ganhando destaque em uma sociedade que enfrenta mudanças econômicas, políticas e sociais. No entanto, os trabalhadores ainda não identificam os sintomas das doenças ocupacionais e quando conseguem, não relatam por temer o desemprego (Girotto \& Diehl, 2016).

A Portaria $\mathrm{n}^{\circ} .1 .339 / 1999$ estabelece os transtornos mentais relacionados ao trabalho: demência, delirium, transtorno orgânico de personalidade e transtorno mental orgânico não especifico, alcoolismo crônico, estresse pós-traumático, neurastenia ou síndrome da fadiga crônica, transtorno do ciclo vigília-sono, síndrome de Burnout e depressão (Brasil, 1999).

Na demência há déficits cognitivos progressivos que comprometem a autonomia nas atividades da vida diária (Sobral \& Paul, 2015). O delirium caracteriza-se por quadros confusionais que comprometem o nível de consciência, o comportamento e o pensamento (Chagas et al., 2016).

Transtornos orgânicos de personalidade e transtorno mental orgânico não específico são comportamentos permanentes diante de uma resposta pessoal e social frente a situações cotidianas e se relaciona à cultura, à forma de pensar, perceber e estabelecer relações interpessoais (Marcon et al., 2018).

$\mathrm{O}$ alcoolismo crônico gera comprometimento dos órgãos do sentido, absenteísmo e/ou abandono do trabalho e predispõe a quadros de ansiedade ou depressão (Palmeira Sobrinho, 2012). O estresse pós-traumático ocorre devido a perturbações psíquicas causadas por situações de estresse no local de trabalho provocada por colegas, clientes ou empregador (Cordeiro et al., 2016). Na neurastenia há fraqueza muscular, dores generalizadas no corpo, fadiga, linfonodos dolorosos, hipertermia e perturbações na memória, atenção e concentração (Zorzanelli et al., 2016). O transtorno do ciclo vigília-sono se expressa por dificuldades em iniciar ou manter o sono, com despertar durante a noite, podendo acarretar transtornos psicossomáticos (fadiga, sonolência diurna, dificuldade de concentração, irritabilidade, ansiedade, depressão e dores musculares) (Neves et al., 2017). Na síndrome de Burnout há despersonalização, falta de realização pessoal, assim como esgotamento físico, mental e emocional que fazem com que o trabalhador tenha dificuldade e descompromisso para trabalhar gerando e aumentando o absenteísmo (Menezeset al., 2017)

Por fim, a depressão relacionada ao trabalho que é alvo desse estudo, é um transtorno de humor que apresenta os seguintes sintomas: emocionais (isolamento social, crises de choro e perda do desejo de realizar as atividades rotineiras), cognitivos (agitação psicomotora, diminuição do rendimento das atividades exercidas), alterações de peso, distúrbio do sono, 
sentimentos de culpada ou inutilidade e até ideação suicida. Esse tipo de depressão reflete do aumento da competitividade das empresas e da globalização, o que se articula aos fatores psicossociais pré-existentes que, atualmente é representado pela falta de apoio interpessoal e por diferentes perdas pessoais e financeiras (Marconiet al., 2018).

De maneira geral, a depressão em trabalhadores é um problema de saúde em todo o mundo e seu aumento se associa aos aspectos que envolvem o cotidiano do trabalho (ambiente, complexidade das relações interpessoais, aumento da competitividade, autonomia excessiva do profissional, entre outros) (Corrêa \& Rodrigues, 2017; Souza \& Andrade, 2018) e isso expressa a importância de se atentar para a depressão no âmbito da saúde do trabalhador, sendo que os casos notificados que resultam de problemas laborais são encaminhados para acompanhamento em serviços públicos de referência em saúde do trabalhador. Essa realidade associada às considerações descritas expressa a importância de se conhecer a prevalência da depressão relacionada ao trabalho para subsidiar estratégias de promoção da saúde mental no trabalhado.

Nessa investigação objetivou-se descrever a ocorrência da depressão relacionada ao trabalho no interior de Minas Gerais e investigar a associação entre depressão relacionada ao trabalho e sexo, idade, situação no mercado de trabalho, tempo de trabalho na ocupação e a categoria da ocupação.

\section{Metodologia}

Estudo retrospectivo, descritivo e quantitativo, aprovado pelo Comitê de Ética da Universidade de Uberaba (parecer: 3.784.697) e desenvolvido em uma unidade pública de Referência em Saúde do Trabalhador do interior de Minas Gerais.

As seguintes variáveis sociodemográficas e epidemiológicas foram avaliadas: sexo, idade, situação no mercado de trabalho, tempo de trabalho na ocupação e categoria profissional.

Foram levantadas as notificações de quadros de depressão relacionada ao trabalho no período de janeiro de 2013 a dezembro de 2019. Pessoas com mais de uma notificação foram incluídas, considerando cada atendimento separadamente. Adotaram-se os seguintes critérios de elegibilidade: apresentar quadros sugestivos de depressão relacionada ao trabalho, conforme exposto na ficha de notificação do Sistema de Informação de Agravos de Notificação, e ter sido ou estar em acompanhamento em um serviço público de Referência em Saúde do Trabalhador. Os critérios de exclusão foram: notificações incompletas e por outras doenças mentais relacionadas ao trabalho.

Os dados foram fornecidos pela Unidade de Referência em Saúde do Trabalhador que possui relatórios anuais das notificações, os quais advêm do serviço de controle de doenças de notificação compulsória e não tem identificação, garantindo o anonimato. Os episódios depressivos foram classificados pelo profissional que atendeu aos trabalhadores na unidade de referência e registrou o CID correspondente na ficha de notificação.

Os dados coletados foram armazenados em planilhas do Excel $^{\circledR}$, sendo as entradas realizadas e checadas e, posteriormente utilizou-se o Bioestat 5.0 para as análises estatísticas descritiva inferencial. Segundo a necessidade, a normalidade foi averiguada por análise visual e pelo teste de Shapiro-Wilk. As associações entre as variáveis foram avaliadas pelo teste Qui-quadrado, satisfeitas as condições para sua aplicação, considerando o nível de significância $\alpha=0,05$.

\section{Resultados}

Foram notificados 52 casos de depressão relacionada ao trabalho, com predomínio de mulheres- 34 (65,38\%). A idade variou de 23 a 67 anos, com mediana de 41,5 anos, primeiro quartil até 34,75 anos e média de 42,08. Entre os trabalhadores do sexo feminino, a idade variou de 23 a 61 anos, mediana de 42,5 anos, primeiro quartil até 36,25 anos e média de 42,68. Dentre os homens, a idade variou de 24 a 67 anos, mediana de 38,5 anos, primeiro quartil até 32,25 anos e média de 40,95.

Os episódios depressivos foram classificados em leve, moderado ou grave, tendo sido avaliada a distribuição dos casos notificados, segundo a classificação do episódio e o sexo do trabalhador (Tabela 1). 
Tabela 1. Distribuição de episódios depressivos relacionados ao trabalho, segundo a classificação do episódio e o sexo.

\begin{tabular}{lccccccc}
\hline Classificação do episódio depressivo relacionado ao trabalho & \multicolumn{2}{c}{ Feminino } & \multicolumn{2}{c}{ Masculino } & \multicolumn{2}{c}{ Total } \\
& $\mathbf{n}$ & $\mathbf{\%}$ & $\mathbf{N}$ & $\mathbf{\%}$ & $\mathbf{n}$ & $\mathbf{\%}$ \\
\hline Episódio depressivo leve & 11 & 21,15 & 5 & 9,62 & 16 & 30,77 \\
Episódio depressivo moderado & 11 & 21,15 & 10 & 19,24 & 21 & 40,39 \\
Episódio depressivo grave & 12 & 23,08 & 2 & 3,84 & 14 & 26,92 \\
Episódio depressivo não especificado & 0 & 0 & 1 & 1,92 & 1 & 1,92 \\
\hline Total & $\mathbf{3 4}$ & $\mathbf{6 5 , 3 8}$ & $\mathbf{1 8}$ & $\mathbf{3 4 , 6 2}$ & $\mathbf{5 2}$ & $\mathbf{1 0 0}$ \\
\hline
\end{tabular}

Fonte: SINAN (2019).

Ao se analisar a gravidade do episódio depressivo (leve, moderado ou grave), independente do sexo do trabalhador, não se encontrou diferença significativa entre as suas frequências $(\mathrm{p}=0.2294)$. Considerando o sexo, também não foi encontrada, nessa amostra, associação com o episódio leve $(\mathrm{p}=0.057)$ e nem com o episódio moderado $(\mathrm{p}=0.8861)$. No entanto, foi encontrada associação do episódio depressivo grave com o sexo feminino ( $\mathrm{p}=0.0004)$. Esses dados sugerem que as mulheres trabalhadoras são mais suscetíveis à depressão grave que os homens.

Avaliou-se a frequência da depressão relacionada ao trabalho, em qualquer classificação (leve, moderada ou grave), de acordo com a situação no mercado de trabalho (Tabela 2). Considerando o regime de contrato, pode-se agrupar os trabalhadores com vínculo celetista (empregado registrado com carteira assinada e servidores públicos celetistas) que somados, correspondem a 55,78\% dos trabalhadores com depressão. Ressalta-se que o grupo de servidores públicos estatutários, apesar de apresentar menor frequência de depressão, compõe quase a metade $(44,22 \%)$ da amostra de trabalhadores deprimidos. Encontrou-se associação $(\mathrm{p}<0.0001)$ entre a depressão e situação no mercado de trabalho, com maior frequência entre os servidores públicos celetistas $(28,85 \%)$, seguidos de empregados registrados com carteira assinada $(26,93 \%)$ e servidores públicos estatutários (19,23\%).

Tabela 2. Frequência de episódios depressivos relacionados ao trabalho de acordo com a situação no mercado de trabalho.

\begin{tabular}{lcc}
\hline Situação no mercado de trabalho & Episódio depressivo relacionado ao trabalho \\
& $\mathbf{N}$ & $\mathbf{\%}$ \\
\hline Empregado registrado com carteira assinada & 14 & 26,93 \\
Autônomo/ conta própria & 4 & 7,69 \\
Servidor público estatutário & 10 & 19,23 \\
Servidor público celetista & 15 & 28,85 \\
Aposentado & 3 & 5,77 \\
Desempregado & 5 & 9,61 \\
Outros & 1 & 1,92 \\
\hline Total & $\mathbf{5 2}$ & $\mathbf{1 0 0}$ \\
\hline
\end{tabular}

Fonte: SINAN (2019).

Ao se analisar as categorias profissionais na amostra, verificou-se uma grande diversidade, optando-se por agrupá-las em duas: profissionais da área de saúde e outros profissionais (Tabela 3). 
A ocorrência de profissionais da área de saúde com depressão relacionada ao trabalho na amostra foi significativamente menor que nas demais categorias profissionais $(\mathrm{p}<0.0001)$. Contudo, a grande diversidade de categorias profissionais na amostra, o pequeno número de trabalhadores da área de saúde no total de notificações e o fato de não se conhecer o universo de profissionais, sejam da área de saúde ou de outras categorias profissionais, impossibilita considerar uma inferência de associação.

Tabela 3. Frequência de episódios depressivos relacionados ao trabalho de acordo com a categoria profissional.

\begin{tabular}{lcc}
\hline Categoria profissional & N & \% \\
\hline Profissionais da área de saúde & 9 & 17,31 \\
Outros profissionais & 43 & 82,69 \\
\hline Total & $\mathbf{5 2}$ & $\mathbf{1 0 0}$ \\
\hline
\end{tabular}

Fonte: SINAN (2019).

Investigou-se também, a frequência dos episódios depressivos relacionados ao trabalho de acordo com o tempo de trabalho na ocupação (Tabela 4).

Tabela 4. Frequência de episódios depressivos relacionados ao trabalho de acordo com o tempo de trabalho na ocupação.

\begin{tabular}{lcc}
\hline Tempo de trabalho na ocupação (anos) & Episódio depressivo relacionado ao trabalho \\
& $\mathbf{N}$ & $\mathbf{\%}$ \\
\hline Até 2 & 18 & 34,62 \\
3 a 6 & 11 & 21,15 \\
7 a 10 & 10 & 19,23 \\
Mais de 10 & 13 & 25,00 \\
\hline Total & $\mathbf{5 2}$ & $\mathbf{1 0 0}$ \\
\hline
\end{tabular}

Fonte: SINAN (2019).

Não foi encontrada associação do tempo de trabalho na ocupação com episódios depressivos relacionados ao trabalho( $\mathrm{p}=0.1313)$, sugerindo que o tempo de permanência na mesma função não contribua para o desenvolvimento desse tipo de depressão.

Ao se analisar a distribuição dos episódios depressivos relacionados ao trabalho de acordo com a faixa etária (Tabela 5), as maiores frequências foram observadas dos 29 aos 46 anos de idade ( $\mathrm{p}<0.0001$ ), cujas frequências somadas correspondem a $50 \%$ das ocorrências de depressão entre os trabalhadores avaliados. 
Tabela 5. Frequência de episódios depressivos relacionados ao trabalho de acordo com a faixa etária.

\begin{tabular}{lcc}
\hline Faixa etária (anos) & Episódio depressivo relacionado ao trabalho & $\%$ \\
& $\mathbf{N}$ & 7.69 \\
23 a 28 & 4 & 17.31 \\
29 a 34 & 9 & 23.08 \\
35 a 40 & 12 & 26.92 \\
41 a 46 & 14 & 7.69 \\
47 a 52 & 4 & 9.62 \\
53 a 58 & 5 & 7.69 \\
59 a 67 & 4 & $\mathbf{1 0 0}$ \\
\hline Total & $\mathbf{5 2}$ & \\
\hline
\end{tabular}

Fonte: SINAN (2019).

\section{Discussão}

Assim como nessa investigação em que 65,38\% das notificações eram de mulheres, em investigação que ocorreu na Bahia e objetivou descrever características dos casos de transtorno mental relacionados ao trabalho notificados no período de 2007 a 2012 também houve predomínio de mulheres (Cordeiro et al., 2016). Achados como esse também ocorreram em investigação desenvolvida no Piauí que objetivou analisar os afastamentos de trabalhadores por transtornos mentais e comportamentais, durante os meses de junho e julho de 2017, sendo que 51,3\% dos registros eram de mulheres (Fernandes et al., 2018). Predomínio da depressão no sexo feminino foi relatado em estudo (Galinari et al., 2020) que abordou depressão entre professores.

Destaca-se que foi encontrada associação da depressão grave com o sexo feminino ( $\mathrm{p}=0.0004)$, assim como em estudo (Leão et al., 2018) que justifica que as mulheres apresentam mais ansiedade que os homens, o que resulta de diversas situações, tais como: conflitos familiares, fatores socioeconômicos, violência e outros. Nesse sentido, acrescenta-se a necessidade de considerar os fatores biológicos e hormonais advindos do período menstrual, parto, puerpério e menopausa (Coutinho et al., 2015). Estudos como o presente, em que diferentes categorias profissionais são contempladas, reforçam os achados nas investigações entre profissões mormente femininas, como a docência na educação infantil. Mostram também que, ainda que algumas divergências possam ser observadas entre autores que discutem a influência de fatores biológicos, sociais e afetivos na depressão sofrida por homens e mulheres, em geral a depressão é mais frequente nelas. E isso não quer dizer que a depressão entre as mulheres trabalhadoras não tenha relação com o trabalho, mas que elas podem sofrer com maior intensidade os fatores relacionados ao trabalho que contribuam para a depressão, quando comparadas aos homens. A associação da depressão grave com o sexo feminino, sinaliza que esse adoecimento está relacionado a fatores extrínsecos e não orgânicos, reforçando o papel de relações insalubres com o trabalho ou no ambiente em que este se desenvolve.

A idade identificada nos resultados dessa investigação (mínima de 23 e máxima de 67 anos, com mediana de 41.5) corroborou ao estudo que identificou que as pessoas que se afastaram por transtornos mentais tinham idade mínima de 19 anos e máxima de 73 anos de (Fernandes et al., 2018). Foi identificada associação dos episódios depressivos com a faixa etária, como apontado em outros estudos. No entanto, na presente investigação a ocorrência de depressão concentrou-se na faixa etária dos 29 aos 46 anos, o que difere de estudos que, apesar de também apontarem existir associação entre a depressão relacionada ao trabalho e a idade, mostram ênfase para os adultos jovens (faixa etária de 25 a 29 anos) (Cordeiro et al., 2016; Oliveira \& Araújo, 2017; Rotta et al., 2016). A ausência de associação dessa variável com episódios depressivos relacionados 
ao trabalho faz pensar se mecanismos protetivos contra a depressão possam ser utilizados igualmente por indivíduos de todas as faixas etárias em nosso universo de estudo ou se os mecanismos protetivos, que porventura sejam desenvolvidos com a idade ou as experiências ao longo da vida, não sejam suficientes frente às relações com o trabalho e no ambiente laboral.

Quanto a situação no mercado de trabalho, identificou-se predomínio daqueles que trabalham em regime celetista (55.78\%) e isso corrobora a investigação (Costa, 2017) que detectou prevalência de 62.5\% de transtornos mentais relacionados ao trabalho entre pessoas que exercem atividade laboral sob este regime de contrato e $18.8 \%$ em servidor público estatutário. No presente estudo, chama atenção a associação da depressão com o regime de trabalho celetista, seja no serviço público ou na iniciativa privada. De maneira geral, o vínculo celetista garante direitos trabalhista perante a Constituição Federal, mas pode favorecer a rotatividade de profissionais nas equipes e dificultar a permanência do colaborador no serviço, ocasionando impacto em sua saúde física e mental (Moreira et al., 2016).

Nesse sentido, nos serviços públicos existem situações que contribuem para o aparecimento de agravos mentais, tais como: ausência de políticas organizacionais estruturadas e de reconhecimento, abuso de poder, má distribuição das tarefas, sobrecarga, problemas de relacionamento interpessoal e doutrina rígida. Neste estudo, os servidores públicos estatutários, apesar de apresentarem menor frequência de depressão relacionada ao trabalho, compõem quase a metade $(44,22 \%)$ da amostra (Silva \& Bueno, 2017). Tal achado sinaliza que se a estabilidade de emprego pode diminuir o risco desse tipo de depressão, pode também facilitar ao trabalhador buscar atendimento com notificação, por não temer a demissão. Seguindo essa linha de pensamento, a depressão relacionada ao trabalho entre os trabalhadores em regime celetista pode estar subnotificada, levando a maior preocupação com aqueles que exercem sua atividade laboral sob esse regime contratual.

Em nossos resultados, o tempo de trabalho na ocupação não se associa à depressão relacionada ao trabalho ( $\mathrm{p}=0.1313$ ), o que difere de outra investigação (Lima et al., 2015), a qual demonstra que a chance de exposição a estressores desencadeadores desse tipo de depressão é tão maior quanto maior for o tempo de trabalho. Seguindo a premissa de que o tempo de trabalho na ocupação equivale a tempo de exposição a fatores que podem desencadear depressão, essa variável está relacionada aos demais fatores que por sua vez dependem da natureza da ocupação. Isso pode explicar a divergência de nossos achados com os de outros autores.

Ao se especificar a ocorrência de transtornos mentais relacionados ao trabalho entre profissionais da área de saúde, verificou-se a existência da depressão foi significativamente menor $(\mathrm{p}<0.0001)$ em comparação com outras categorias profissionais, o que difere de outro achado (Corrêa \& Rodrigues, 2017), onde a prevalência de depressão na área de saúde é maior, devido à sobrecarga de trabalho, desvalorização do trabalho, longas jornadas de trabalho e pressão da chefia. Acreditase que essa disparidade de resultados se justifica pelo desconhecimento do total de trabalhadores da área de saúde e de outras categorias profissionais, impossibilitando inferir associação.

No presente estudo, características dos dados, como o pequeno número de trabalhadores da área de saúde, impossibilitaram considerar a associação com verdadeiro. No entanto, mesmo sem inferir associação, destaca-se que em estudo realizado com 86 profissionais de saúde de Minas Gerais, identificou-se uma prevalência de depressão relacionada ao trabalho maior entre profissionais de enfermagem (40.7\%) (Pereira et al., 2017) e em estudo realizado em Pernambuco com 200 profissionais de saúde, verificou-se que $23 \%$ dos participantes apresentavam quadro depressivo leve e $7.5 \%$ moderado, sendo que ambos se relacionavam ao trabalho (Sampaio et al., 2020).

\section{Conclusão}

Ainda que a subnotificação seja um fator limitante para estudos dessa natureza, os dados obtidos mostram associação da depressão grave com o sexo feminino, sinalizando que o adoecimento seja, de fato, relacionado a fatores extrínsecos e não orgânicos, reforçando o papel de relações insalubres com o trabalho ou no ambiente em que este se desenvolve. No período 
investigado, a faixa etária dos trabalhadores com depressão foi bastante ampla, sugerindo que mecanismos protetivos que porventura sejam desenvolvidos com a idade ou as experiências ao longo da vida, não sejam suficientes frente às relações com o trabalho e no ambiente laboral, ou que esses mecanismos sejam utilizados igualmente em qualquer idade. Considerando a situação no mercado de trabalho, a associação dos estados depressivos com o vínculo celetista, seja no serviço público ou na iniciativa privada, indica que a instabilidade na relação contratual seja um fator de risco. Os resultados obtidos evidenciam a importância do tema e a necessidade de medidas protetivas à saúde mental do trabalhador.

\section{Referências}

Brasil. (1999). Portaria $n^{\circ} 1.339$, de 18 de novembro de 1999. Ministério da Saúde.

Chagas, N. M. S., Borges, D. G. S., \& Chagas, M. H. N. (2016). Delirium as risk factor for dementia in older adults: an update. Jornal Brasileiro de Psiquiatria, 65(1), 94-98.

Cordeiro, T. M., Mattos, A. I., Cardoso, M. C., Santos, K. O, \& Araújo, T. M. (2016). Reporting of work-related mental disorders among workers in Bahia: a descriptive study, 2007-2012. Epidemiologia \& Serviços de Saúde, 25(2), 363-372.

Corrêa, C. R., \& Rodrigues, C. M. L. (2017). Depressão e trabalho: revisão da literatura nacional de 2010 e 2014. Negócios em Projeção, 8(1), 65-74.

Costa, S. S. (2017). Perfil epidemiológico dos transtornos mentais relacionados ao trabalho na região nordeste do Brasil, no período de 2007 a 2016. Artigo (Especialização Saúde Mental e Atenção Básica) - Escola Bahiana de Medicina e Saúde Pública, Salvador, BA.

Coutinho, M. E. M., Giovanini, M., Pavini, L. S., Ventura, M. T., Elias, R. M., \& Silva, L. M. (2015). Aspectos biológicos e psicossociais da depressão relacionado ao gênero feminino. Revista Brasileira de Neurologia Psiquiatria, 19(1), 49-57.

Fernandes, M. A., Santos, J. D. M., Moraes, L. M. V., Lima, J. S. R., Feitosa, C. D. A., \& Sousa, L. F. C. (2018). Mental and behavioral disorders in workers: a study on work leave. Revista da Escola de Enfermagem USP, 52, e03396.

Galinari, P. C., Castro, J. M., Martins, R. E. C., Alencar, N. P. F. C., Azevedo, M. A., Oliveira, T. V. C., Proti, E. S., Araújo, D. A., Guerra, C. H. W., \& Costa,W. J. T. (2020). Depression among teachers: integrative literature review. Revista Eletrônica Acervo Saúde, 2 , e2546.

Girotto, C., \& Diehl, L. (2016). Mental health and work: a reflection on possible relationship between the diagnosis and working conditions. Polem!ca, 16(2), 90-115.

Leão, A. M., Gomes, I. P., Ferreira, M. J. M., \& Cavalcanti, L. P. G. (2018). Prevalence and factors associated with depression and anxiety among university students in the field of health in a large urban center in the northeast of Brazil. Revista Brasileira de Educação Médica, 42(4), 55-65.

Lima, E. P., Assunção, A. A., \& Barreto, S. M. (2015). Prevalence of depression among firefighters. Cadernos de Saúde Pública, 31 (4), 733-743.

Marconi, E. V. N., Etapechusk, J., \& Campos, L. C. A. (2018). Transtornos mentais de personalidade e clínicos; uma breve discussão teórica. Psicologia.PT, $1-13$

Menezes, P. C. M., Alves, E. S. R. C., Aráujo Neto, S. A., Davim, R. M. B., \& Guaré, R. O. (2017). Burnout syndrome: a reflective analysis. Journal of Nursing UFPE on line, 12(11), 5092-5101.

Moreira, I. J. B., Horta, J. A., Duro, L. N., Borges, D. T., Cristofari, A. B., Chaves, J., Bassani, D. C. H., Cerizolli, E. D., \& Teixeira, R. M. (2016). Sociodemographic and occupational profile and evaluation of mental health disorders of employees of the Family Health Strategy in a city of Rio Grande do Sul, RS. Revista Brasileira de Medicina de Família e Comunidade, 11(38), 1-12.

Neves, G. S. M. L., Macedo, P., \& Gomes, M. M. (2017). Sleep disorders: up to date (1/2). Revista Brasileira de Neurologia, 53(3), 19-30.

Oliveira, A. M. N., \& Araujo, T. M. (2017). Situations of imbalancing between stress-rewards and common mental disorders in basic health care workers. Trabalho, Educação \& Saúde, 16(1), 243-262.

Palmeira Sobrinho, Z. (2012). O alcoolismo e os seus reflexos sociojurídicos sobre as relações de trabalho. Revista Fórum Trabalhista, 1(1), $167-191$.

Pereira IF. (2017) Depression and use of medicines in nursing personnel. Arquivos de Ciências da Saúde, 24(1):70-4.

Rotta, D. S., Pinto, M. H., Lourencao, L., \& Teixeira, P. R. (2016). Anxiety and depression levels among multidisciplinary health residents. Revista Rene, $17(3), 372-377$

Sampaio, L. R., Oliveira, L. C., \& Pires, M. F. D. N. (2020). Empathy, depression, anxiety and stress in Brazilian Health Professionals. Ciências Psicológicas, 14(2), e2215.

Silva, R. B., \& Bueno, H. P. V. (2015). A saúde mental e os principais motivos de afastamento do servidor público brasileiro. Artigo (Especialização em Saúde Mental) - Universidade Católica Dom Bosco, Campo Grande, MS.

Sobral, M., \& Paúl, C. (2015). Reserva cognitiva, envelhecimento e demências.Revista E-Psi,5(1), 113-134. 
Research, Society and Development, v. 10, n. 9, e18510917759, 2021

(CC BY 4.0) | ISSN 2525-3409 | DOI: http://dx.doi.org/10.33448/rsd-v10i9.17759

Souza, D. A. L., \& Andrade, E. G. S. (2018). Quality of life of nursing professionals: factors influencing depression atwork. Revista de Iniciação Científica e Extensão, 1(2), 57-66.

Teixeira, L. C., \& Vieira, A. M. (2018). Síndrome de Burnout: um estudo de caso sobre a exaustão emocional e o estresse ocupacional de uma servidora pública em um município do estado de Minas Gerais. Cadernos Científicos FAGOC Graduação e Pós-Graduação, 3(2), $36-43$.

Vitorino, M. F., Rodrigues, M. S. D., Evangelista, C. B., Guimarães, K. S. L., Batista, J. B. V., Fonsêca, A. G. S., Aráujo, A. L. B., \& Melo, F. M. A. B. (2018). Burnout syndrome: knowledge of neonatal nursing team. Journal of Nursing UFPE on line, 9(12), 2308-2314.

Zorzanelli, R., Vieira, I., \& Russo, J. A. (2016). Several names for tiredness: emergent categories and their relationship with the world of work. Interface Comunicação Educação Saúde, 20(56), 77-88. 